\title{
FORTSCHRITTE DER
}

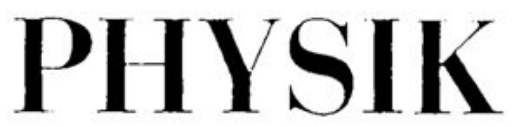

HeRAUSgegebeN IM AUFTRAGE DER PHYSIKALISCHEN GESFLLSCHAFT DER

DEUTSCHEN DEMOKRATISCHEN RIPUBLIK

VON F. KASCHLLHN A. LÖSCHF, R. RITSCHL IND R. ROUPE

HEFT $11 / 12 \cdot 1979 \cdot$ BAND 27

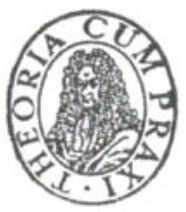

A K A D E M I E - V E R L A G D E R L I N

EVP $20,-M$

31728 


\section{BEZUGSMÖGLICHKEITEN}

Bestellungen sind zu richten

- in der DDR an das Zeitungsvertriebsamt, an eine Buchhandlung oder an den AKADEMIE-VERLAG, DDR - 108 Berlin, Leipziger Straße 3-4

- im sozialistischen Ausland an eine Buchhandlung für fremdsprachige Literatur oder an den zuständigen Postzeitungsvertrieb

- in der BRD und Westberlin an eine Buchhandlung oder an die Auslieferungsstelle KUNST UND WISSEN, Erich Bieber, 7 Stuttgart 1, Wilhelmstraße 4-6

- in Österreich an den Globus-Buchvertrieb, 1201 Wien, Höchstädtplatz 3

- in den übrigen westeuropäischen Ländern an eine Buchhandlung oder an die Auslieferungsstelle KUNST UND WISSEN, Erich Bieber GmbH, CH - 8008 Zürich/Schweiz, Dufourstraße 51

- im übrigen Ausland an den Internationalen Buch- und Zeitschriftenhandel; den Buchexport, Volkseigener Außenhandelsbetrieb der Deutschen Demokratischen Republik,

DDR - 701 Leipzig, Postfach 160, oder

an den AKADEMIE-VERLAG,

DDR - 108 Berlin, Leipziger Straße 3-4

Zoitschrift „Fortsehritte der Physik“

Herausgeber: Prof. Dr. Frank Kasehluhn, Prof. Dr. Artur Losche, Prof. Dr. Rudolf Ritochl, Prof. Dr. Robert Rompe, im Auftrag der Physikalischen Gesellschaft der Deutschen Demokratischen Republik.

Verlag: Akademie-Verlag, DDR - 108 Berlin, Leipziger Straße 3-4; Fernruf: 2236221 und 22 36 229; Telex-Nr. 114420; Bank: Staatsbank der DDR, Berlin, Konto-Nr. 6836-26-20712.

Chefredakteur: Dr. Lutz Rothkirch.

Ansebrift der Redaktion: Sektion Physik der Humboldt-Universität zu Berlin, DDR - 104 Berlin, Hessische Straße 2.

Veröffentlicht unter der Lizenznummer 1324 des Presseamtes beizn Vorsitzenden des Ministerrates der Deutschen Demokratischen Republik.

Gesamtherstellung: VEB Druckhaus „Maxim Gorki“, DDR - 74 Altenburg, Carl-von-Osejetzky-StraBe 30/31.

Erscheinungsweise: Die Zeitschrift „Fortschritte der Physik“ erseheint monatlich. Die 12 Hefte eines Jahres bilden einen Band. Bezugspreis je Band 180, - M zuzüglich Versandspesen (Preis für die DDR: 120, - M). Preis je Heft 15, - M (Preis für die DDR: $10,-\mathrm{M})$

Bestellnummer dieses Heftes: 1027/27/11/12.

(c) 1979 by Akademie-Verlag Berlin. Printed in the German Democratic Republic.

AN (EDV) 57618 